\title{
Troponin I in predicting cardiac or pulmonary complications and outcome in subarachnoid haemorrhage
}

\author{
W J Schuiling, P J W Dennesen, J Th J Tans, L M Kingma, A Algra, G J E Rinkel
}

J Neurol Neurosurg Psychiatry 2005;76:1565-1569. doi: 10.1136/jnnp.2004.060913

See end of article for authors' affiliations

......................

Correspondence to:

Dr W J Schuiling,

Department of Neurology; Medical Centre

Leeuwarden, PO Box 888

8901 BR Leeuwarden, The

Netherlands; wjschuiling@ planet.nl

Received

15 December 2005

Revised version received

9 March 2005

Accepted 21 March 2005
Background: Patients with aneurysmal subarachnoid haemorrhage (SAH) are at risk of cardiac and pulmonary complications. Troponin I (cTnl), a reliable marker of myocardial injury, is frequently raised after SAH.

Aims: To investigate the additional value of (cTnl) in predicting cardiac or pulmonary complications and outcome in patients with SAH.

Methods: Admission cTnl was measured in a prospective series of patients admitted within 24 hours of $\mathrm{SAH}$. By means of univariate and multivariate logistic regression models the additional prognostic value of raised cTnl ( $>0.3 \mu \mathrm{g} /$ litre) was investigated compared with established prognosticators (clinical condition on admission, age, and amount of blood on admission computed tomography) for predicting the occurrence of pulmonary oedema, pulmonary gas exchange abnormalities, rhythm disturbances, inadequate cardiac performance, a combination of these complications, and poor outcome. Area under the operator characteristic curve (AUC-ROC) was used to assess additional prognostic value.

Results: Abnormal cTnl concentrations were found on admission in 35 of 68 patients. Abnormal cTnl concentrations and poor clinical condition independently predicted cardiac or pulmonary complications. After extending the model with World Federation of Neurological Surgeons scale and age in addition to abnormal cTnl, the AUC-ROC improved from 0.70 (95\% confidence interval (Cl), 0.57 to 0.83 ) to 0.83 (95\% Cl, 0.72 to 0.93). Abnormal cTnl also independently predicted poor outcome. The additional prognostic value of cTnl for poor outcome is limited.

Conclusions: cTnl measurement is a powerful predictor for the occurrence of pulmonary and cardiac complications, but does not carry additional prognostic value for clinical outcome in patients with aneurysmal SAH.
$\mathrm{S}$ ubarachnoid haemorrhage (SAH) from an intracranial aneurysm remains a devastating event. Approximately $30-50 \%$ of patients who reach the hospital alive die and another $10-20 \%$ remain dependent on help for daily activities. ${ }^{1}$ The leading causes of death and disability are the initial or repeated bleedings, and delayed ischaemic events. ${ }^{2}$ The initial and repeated bleedings may induce extracranial sequelae, of which cardiac and pulmonary complications are the most frequent. ${ }^{3}$ These cardiac and pulmonary complications related to $\mathrm{SAH}$ can lead to disturbed oxygenation of the brain, and thereby contribute to poor outcome in these patients. The excessive catecholamine release after SAH is probably the driving force of left ventricular dysfunction of the heart and pulmonary oedema. ${ }^{4}$ The severity of the brain injury is strongly related to the occurrence of these cardiac and pulmonary complications. ${ }^{5}$ Troponin I (cTnI), a regulatory protein highly specific for cardiac muscle, is a reliable marker of myocardial injury leading to left ventricular dysfunction. ${ }^{6}$ A raised cTnI concentration is a marker of poor prognosis in patients with unstable cardiac ischaemia and also in patients with septic shock. ${ }^{78}$ In recent studies, $30-40 \%$ of patients with SAH showed increased concentrations of cTnI on admission. ${ }^{9}{ }^{10}$ Increased cTnI proved to be a highly sensitive and specific marker for cardiac dysfunction in patients with SAH also. ${ }^{11}{ }^{12}$

Despite the direct link between SAH and cardiac injury, as defined by raised cTnI values, it is not clear whether raised cTnI concentrations on admission independently predict secondary cardiac or pulmonary complications and outcome. Therefore, we performed a prospective study on the additional value of cTnI over established prognosticators (clinical condition on admission, age, and amount of extravasated blood $)^{13} 14$ in predicting cardiac or pulmonary complications and outcome in patients with SAH.

\section{MATERIAL AND METHODS}

Patients

We studied 68 patients with aneurysmal SAH who were treated in the Medical Centre Haaglanden, the Netherlands. All patients with SAH are treated at our intensive care unit for at least two weeks. The diagnosis aneurysmal SAH was based on typical history, a computed tomography (CT) scan showing blood in the basal cisterns and an aneurysm on angiography, or an aneurysmal pattern of haemorrhage on CT in those patients in whom intra-arterial angiography was not performed because of a poor clinical condition from the outset. To be included, patients had to be admitted to the intensive care unit (ICU) within 24 hours after the onset of the haemorrhage. We did not exclude patients with a history of previous cardiac disease. Only one patient with previous stable cardiac disease was included in our study. Patients were monitored in the ICU for two weeks. Three months after $\mathrm{SAH}$ onset a follow up visit for all patients was planned. The

Abbreviations: AUC, area under the curve; $\mathrm{Cl}$, confidence interval; CPIS, clinical pulmonary infection score; CT, computed tomography; cTnl, cardiac troponin I; ECG, electrocardiogram; $\mathrm{FiO}_{2}$, fraction of inspired oxygen; ICU, intensive care unit; MAP, mean arterial pressure; $\mathrm{PaO}_{2}$, partial pressure of oxygen in arterial blood; $\mathrm{ROC}$, operator characteristic curve; SAH, subarachnoid haemorrhage; SIRS, systemic inflammatory response syndrome; WFNS, World Federation of Neurological Surgeons 
Table 1 Patient characteristics

\begin{tabular}{|c|c|}
\hline Characteristic & $\mathbf{N}$ \\
\hline Total number & 68 \\
\hline Women & $53(78 \%)$ \\
\hline Poor condition on admission (WFNS IV-V) & $32(47 \%)$ \\
\hline \multicolumn{2}{|l|}{ Amount of extravasated blood (Hijdra score) } \\
\hline Median ventricular score (range) & $3(0-12)$ \\
\hline Median cisternal score (range) & $20(3-30)$ \\
\hline \multicolumn{2}{|l|}{ Treatment of aneurysm } \\
\hline Clipping & $21(31 \%)$ \\
\hline Coiling & $22(32 \%)$ \\
\hline None & $25(37 \%)$ \\
\hline Median troponin I concentration (range) in $\mu \mathrm{g} / \mathrm{l}$ & $0.4(0-85.2)$ \\
\hline Abnormal troponin I & $35(52 \%)$ \\
\hline \multicolumn{2}{|l|}{ Outcome events } \\
\hline Pulmonary oedema & $19(28 \%)$ \\
\hline $\mathrm{PaO}_{2} / \mathrm{FiO}_{2}$ ratio $\leqslant 200$ & $25(37 \%)$ \\
\hline Rhythm disturbances & $11(16 \%)$ \\
\hline Inadequate cardiac performance & $17(25 \%)$ \\
\hline \multicolumn{2}{|l|}{ Predefined outcome } \\
\hline Cardiopulmonary complications* & $39(57 \%)$ \\
\hline Poor clinical outcome & 40 (59\%) \\
\hline \multicolumn{2}{|c|}{$\begin{array}{l}\text { *Defined as pulmonary oedema or a } \mathrm{PaO}_{2} / \mathrm{FiO}_{2} \text { ratio } \leqslant 200 \text {, or the } \\
\text { occurrence of a rhythm disturbance or an inadequate cardiac } \\
\text { performance. } \\
\mathrm{FiO}_{2} \text {, fraction of inspired oxygen; } \mathrm{PaO}_{2} \text {, partial pressure of oxygen in } \\
\text { arterial blood; WFNS, World Federation of Neurological Surgeons. }\end{array}$} \\
\hline
\end{tabular}

initial 10 patients were included non-consecutively; the remaining 58 patients were included consecutively between June 2002 and February 2004.

\section{Baseline measurements}

The neurological condition on admission was assessed by means of the World Federation of Neurological Surgeons (WFNS) scale. ${ }^{15}$ WFNS grades I, II, and III were defined as a good neurological condition and WFNS grades IV or $\mathrm{V}$ as a poor neurological condition. Because the relation between age and cardiac complications is probably non-linear, we analysed age in tertiles. The risk implications were similar in the second and third tertiles and higher than in the first; therefore, we dichotomised between the first and second tertiles. The amount of cisternal and ventricular blood was scored according to the Hijdra score. ${ }^{16}$ The sum scores of blood in the cisterns (range, 0-30) and ventricles (range, 012) were dichotomised at their median value. Blood samples were obtained within 24 hours of SAH onset to assess cTnI. Venous blood was collected at our emergency department and repeated later at the ICU. No serial measurements after the first 24 hours were performed for the patients in our study. In general, cTnI is undetectable in normal volunteers. It was measured using an AxSYM random access immunoanalyser (Abbott Laboratories, Maidenhead, Berkshire, UK) The lower limit of detection was $0.3 \mu \mathrm{g} /$ litre, so that abnormal values were defined as higher than $0.3 \mu \mathrm{g} /$ litre.

Table 2 Univariate analysis of predictors for cardiopulmonary complications

\begin{tabular}{ll}
\hline Variable & OR $(95 \% \mathrm{Cl})$ \\
\hline Troponin I $>0.3 \mu \mathrm{g} / \mathrm{I}$ & $11.9(3.7$ to 39$)$ \\
WFNS $\geqslant 4$ & $3.3(1.2$ to 9.1$)$ \\
Age $>51$ years & $2.9(1.0$ to 8.6$)$ \\
Amount of extravasated blood (Hijdra & \\
score) & \\
$\quad$ Ventricular score $>3$ & $2.1(0.8$ to 5.8$)$ \\
$\quad$ Cisternal score $>20$ & $1.3(0.5$ to 3.6$)$ \\
\hline
\end{tabular}

*Dichotomised between the first and second tertiles. $\mathrm{Cl}$, confidence interval; OR, odds ratio; WFNS, World Federation of Neurological Surgeons.
A chest $x$ ray was obtained on admission and repeated every two days during the first two weeks of hospitalisation. These chest $x$ rays were scored by a radiologist, who was blinded to the patient's clinical information and cTnI, as follows: grade 0 , no alveolar consolidation; grade 1 , little alveolar consolidation; grade 2, severe alveolar consolidation; and grade 3, diffuse bilateral alveolar consolidation. We categorised grades 2 and 3 as the occurrence of pulmonary oedema. For each patient we continuously recorded the following data during the first two weeks of admission: heart rate and rhythm; systolic, diastolic, and mean arterial blood pressure (MAP); fraction of inspired oxygen $\left(\mathrm{FiO}_{2}\right)$; and partial pressure of oxygen in arterial blood $\left(\mathrm{PaO}_{2}\right)$. Pulmonary gas exchange was expressed as the $\mathrm{PaO}_{2} / \mathrm{FiO}_{2}$ ratio. Comparable to patients with an adult respiratory distress syndrome, ${ }^{17}$ a $\mathrm{PaO}_{2} / \mathrm{FiO}_{2}$ ratio of $\leqslant 200 \mathrm{~mm} \mathrm{Hg}$ was defined as a severe disturbance of pulmonary gas exchange. The clinical pulmonary infection score (CPIS) was used in the diagnosis of pneumonia. ${ }^{18}$ The CPIS is a combination of clinical, radiographical, and microbiological criteria, with a maximum score of 12 . Values of 6 or higher are considered diagnostic for pneumonia. Patients with a CPIS score of 6 or higher were excluded from the analysis regarding pulmonary oedema and abnormalities in pulmonary gas exchange. A systemic inflammatory response syndrome (SIRS) was considered present when $\geqslant 2$ of the following conditions were met: temperature of $<36^{\circ} \mathrm{C}$ or $>38^{\circ} \mathrm{C}$, heart rate of $>90$ beats/minute, respiratory rate of $>20$ breaths/minute or a $\mathrm{PCO}_{2}<32 \mathrm{~mm} \mathrm{Hg}$, and white blood cell count of $<410^{9}$ /itre or $>12 \times 10^{9}$ /itre. ${ }^{19}$

Cardiac rhythm disturbances (atrial fibrillation or supraventricular tachycardia) in which anti-arrhythmic treatment was needed were recorded. Inadequate cardiac performance was defined as the need for inotropic support to obtain a MAP $>65 \mathrm{~mm} \mathrm{Hg}$ in the first four days after admission. An admission 12 lead electrocardiogram (ECG) of each patient was analysed by an experienced electrocardiographer, who was blinded to the patient's condition. ST depression greater than $0.1 \mathrm{mV}$ in limb leads or $0.2 \mathrm{mV}$ in precardial leads was defined as an abnormal finding. A $\mathrm{T}$ wave was defined as inverted if at least $0.1 \mathrm{mV}$ in depth. $\mathrm{T}$ wave abnormalities were assessed in leads I, II, aVL, aVF, and $\mathrm{V}_{2-6}$. We defined ischaemic-like ECG abnormalities as the presence of ST depression or T wave inversion, or both, in at least two leads. All patients received standard intensive care treatment, which included administration of oral nimodipine, an anticonvulsant agent, and intravenous fluids to maintain normovolaemia. In case of hypotension, the nimodipine dose was reduced or even postponed before inotropic support was started. For the prevention of ventilator associated pneumonia, all mechanically ventilated patients received selective decontamination of the digestive tract. ${ }^{20}$ Surveillance cultures were taken on admission and subsequently every other day of the oropharynx, trachea, and rectum. Two blood cultures were drawn if the rectal temperature exceeded $38.5^{\circ} \mathrm{C}$.

\section{Definition of outcome events}

The predefined outcomes were the occurrence of secondary cardiac or pulmonary complications and poor clinical outcome.

A cardiac or pulmonary complication was defined as the occurrence of at least one of the aforementioned complications (pulmonary oedema, abnormalities in pulmonary gas exchange, rhythm disturbances, or an inadequate cardiac performance).

Poor outcome was defined as a modified Rankin scale score of 4 or 5 (that is, dependent on activities in daily life) or death three months after onset. ${ }^{21}$ 
Table 3 Multivariate analysis of predictors for cardiopulmonary complications

\begin{tabular}{|c|c|c|c|c|}
\hline & \multicolumn{2}{|c|}{ Baseline characteristics* } & \multicolumn{2}{|c|}{ Baseline characteristics and an abnormal cTnl } \\
\hline & OR & $95 \% \mathrm{Cl}$ & OR & $95 \% \mathrm{Cl}$ \\
\hline WFNS $\geqslant 4$ & 3.3 & 1.2 to 9.1 & - & \\
\hline Age $>51$ year & 3.0 & 0.98 to 9.2 & 4.1 & 1.1 to 15.8 \\
\hline Troponin $I \geqslant 0.3 \mu \mathrm{g} / \mathrm{I}$ & & & 14.1 & 3.9 to 51 \\
\hline AUC of ROC & 0.70 & 0.57 to 0.83 & 0.83 & 0.72 to 0.93 \\
\hline
\end{tabular}

\section{Data analysis}

We related the occurrence of a cardiac or pulmonary complication to baseline characteristics (WFNS, amount of blood on baseline CT, and age) and to an abnormal cTnI in univariate and multivariate analyses by means of logistic regression models, yielding crude odds ratios. In the multivariate analysis, we developed a model with forward selection based on the baseline characteristics, with a corresponding $p$ value of $<0.10$ in the univariate analysis. We extended this model with data on cTnI. We evaluated the discriminatory power of the models with the area under the curve (AUC) of the corresponding receiver operator characteristic curve (ROC). ${ }^{22}$ An AUC can range from 0.50 (no discriminatory power) to 1.0 (perfect prediction). We categorised cTnI values in tertiles to assess a possible "dose response" effect of the cTnI values and the occurrence of a cardiac or pulmonary complication. A similar strategy was used for the analysis of poor outcome after three months. A secondary analysis for poor outcome and secondary complications was performed with the 58 consecutive patients (10 non-consecutive patients excluded).

\section{RESULTS}

A raised cTnI concentration (range, 0.3-85.2 $\mu \mathrm{g} / \mathrm{litre}$ ) was found in 35 of the 68 patients on admission to the ICU. Table 1 provides the clinical characteristics of the patients. Signs of cardiac ischaemia on ECG (presence of ST depression or T wave inversion, or both, in at least two leads) occurred in six of 30 patients without abnormal cTnI and 17 of 33 with abnormal cTnI. In five patients the admission ECG could no longer be retrieved.

\section{Cardiac and pulmonary complications}

An abnormal cTnI was found on admission in 16 of the 19 patients with pulmonary oedema, 20 of 25 patients with a disturbance of pulmonary gas exchange, nine of 11 patients with rhythm disturbances, and 13 of 17 patients with inadequate cardiac performance. SIRS was present in 56 patients, including all 17 patients with inadequate cardiac performance. In the patients with inadequate cardiac performance, no pulmonary infiltrate was detected on the chest $x$ ray. In eight of these patients, blood cultures were set up and were positive in two patients. Both blood cultures yielded coagulase negative staphylococci. In 24 of the remaining 29 patients with SIRS blood cultures were set up; in four patients these were positive for coagulase negative staphylococci.

A cardiac or pulmonary complication was seen in 10 of 33 patients with an undetectable cTnI and in 29 of 35 patients with a raised cTnI. Two patients were excluded from the analysis regarding pulmonary oedema because of pneumonia. In four patients, pulmonary infiltrates were noted on chest $x$ rays during their stay in the ICU. These patients had no clinical signs of pneumonia and no potential pathogen was cultured from tracheal secretions or blood (CPIS score was below 6 in all four patients). These patients were included in the analysis; an additional analysis without these four patients yielded similar results.

An abnormal cTnI and a WFNS score $\geqslant 4$ on admission predicted the occurrence of cardiac or pulmonary complications in univariate analysis (table 2). We did not find an increasing risk for complications with increasing concentrations of cTnI. Patients in the highest tertile of cTnI values (3.3-85.2 $\mu \mathrm{g} /$ litre) had a similar risk increase of cardiopulmonary complications (odds ratio, 11.5; 95\% confidence interval (CI), 3.0 to 44 ) as those in the middle tertile (odds ratio, $14.1 ; 95 \% \mathrm{CI}, 2.6$ to 76 ), both in comparison with the lowest tertile. In the model with established baseline characteristics, the WFNS score and age were retained in multivariate modelling (corresponding $\mathrm{p}$ value was $<0.10$ in univariate analysis). Upon extension of this basic model with abnormal cTnI, this last variable and age $>51$ years remained in the model. This result shows that cTnI is an independent prognosticator for cardiopulmonary complications. The AUC improved from 0.70 (95\% CI, 0.57 to 0.83 ) for the basic model to 0.83 (95\% CI, 0.72 to 0.93 ) with extension of cTnI (table 3). Secondary analyses for cardiopulmonary complications in the 58 consecutive patients (10 nonconsecutive patients excluded) produced similar results (AUC of the ROC improved from 0.68 (95\% CI, 0.54 to 0.83 ) for the basic model to 0.85 ( $95 \%$ CI, 0.75 to 0.95 ) with extension of $\mathrm{cTnI})$.

\section{Poor outcome}

In the univariate analysis, both an abnormal cTnI and all established baseline characteristics were strong predictors of poor outcome (table 4). An abnormal cTnI was an independent prognosticator for poor outcome in the multivariate model. The AUC of the ROC did not improve with extension of an abnormal CTnI $(0.89 ; 95 \%$ CI, 0.81 to 0.97$)$ to the model based on these established baseline characteristics (0.86; $95 \%$ CI, 0.77 to 0.95 ). We did not find an increasing risk for poor outcome with increasing tertiles of cTnI. Secondary analyses for poor outcome with the 58 consecutive patients ( 10 nonconsecutive patients excluded) produced similar results (AUC of the ROC did not improve with extension of an abnormal CTnI $(0.90 ; 95 \%$ CI, 0.82 to 0.98$)$ to the model based on the established characteristics $(0.88 ; 95 \% \mathrm{CI}, 0.79$ to 0.97$))$.

\section{DISCUSSION}

An abnormal cTnI concentration is a powerful predictor for the occurrence of pulmonary and cardiac complications in patients with aneurysmal SAH. In our series, we found a high frequency of pulmonary oedema, disturbances of pulmonary gas exchange, rhythm disturbances, and an inadequate cardiac performance. Abnormal cTnI was significantly related to all these complications. A raised CTnI was also an independent prognosticator for the occurrence of a poor outcome. However, the additional prognostic information of cTnI for poor outcome is limited. The severity of brain injury 
Table 4 Logistic regression of predictors for poor outcome*

\begin{tabular}{|c|c|c|c|c|}
\hline & \multicolumn{2}{|c|}{ Univariate } & \multicolumn{2}{|c|}{ Multivariate } \\
\hline & OR & $95 \% \mathrm{Cl}$ & OR & $95 \% \mathrm{Cl}$ \\
\hline Abnormal troponin I & 3.9 & 1.4 to 11 & 4.7 & 1.1 to 20 \\
\hline WFNS $\geqslant 4$ & 9.9 & 3.0 to 31 & 4.9 & 1.1 to 23.4 \\
\hline Age $>51$ year & 4.7 & 1.6 to 14.2 & 6.3 & 1.3 to 30.7 \\
\hline \multicolumn{5}{|c|}{ Amount of extravasated blood (Hijdra score) } \\
\hline Ventricular score $>3$ & 12.5 & 3.2 to 48 & 8.1 & 1.5 to 42 \\
\hline Cisternal score $>20$ & 2.9 & 1.0 to 7.8 & - & \\
\hline AUC of ROC & & & 0.89 & 0.81 to 0.97 \\
\hline
\end{tabular}

is the leading cause of a poor outcome, ${ }^{2}$ and pulmonary and cardiac complications are less important factors for the eventual outcome. The minor importance of pulmonary and cardiac complications for eventual outcome probably explains why a factor that predicts the occurrence of these complications does not have additional prognostic value for the eventual outcome.

In contrast to previous studies, we assessed the additional prognostic value of cTnI to established prognosticators for pulmonary and cardiac complications, and for clinical outcome. The proportion of patients with a raised troponin value in our patient group is larger than in other recent studies. This larger proportion is probably explained by the large fraction of patients with a poor clinical condition on admission, the short interval between onset of SAH and blood sampling, and the large proportion of women in our study. Female sex and shorter time from SAH onset have been associated with higher concentrations of cTnI. ${ }^{9}$ A possible limitation of our relatively small study is that some patients were not included consecutively. We included these patients at random. An additional analysis without these patients yielded similar results; therefore, we do not think that the inclusion of these patients has biased our results to any great extent.

The release of catecholamines is linked to myocardial necrosis and increases in cardiac enzymes. ${ }^{23} 24$ The cTnI enzyme is a specific and highly sensitive marker of cardiac injury. The release of catecholamines can also cause pulmonary oedema by means of increasing transmural pulmonary vascular pressures caused by a combination of $\alpha$ and $\beta$ adrenoceptor activation, and cardiac injury. ${ }^{525}$ These complications are responsible for hypotension and hypoxia, two important secondary sequelae that influence outcome after acute brain injury. Although four patients might have been misclassified as having pulmonary oedema and not pneumonia, this did not influence the relation between abnormal cTnI and pulmonary oedema. In addition, it can be argued that a decrease in MAP was related to nimodipine, a potent vasodilator, especially when give intravenously. This seems unlikely in our patients who received only oral nimodipine. Oral nimodipine has been shown to decrease MAP in a minority of patients compared with intravenous administration. ${ }^{26}$ Finally, the possibility of septic shock and not cTnI being related to a decrease in MAP should be considered. Coagulase negative staphylococci were cultured in two of 17 patients with inadequate performance and in four patients who did not require inotropic support. Despite the fact that coagulase negative staphylococci pose a lower risk of causing septic shock than other organisms in the ICU, an effect on MAP cannot be excluded entirely. ${ }^{27}$

We found three other studies that mentioned an effect on outcome of cTnI in patients with SAH. In a study of 47 patients (eight with increased cTnI), a worse clinical outcome was suggested in patients with a raised cTnI, but the results were not significant. $^{28}$ In another study comprising 43 patients, a worse clinical outcome (death or discharge to nursing home) was seen in patients with a raised cTnI (relative risk, 1.8; 95\% CI, 0.7 to 4.8 ) but the results were not significant. ${ }^{12}$ A recent study mentioned a strong association between cTnI and outcome in univariate analysis (relative risk, $2.4 ; 95 \% \mathrm{CI}, 1.7$ to 3.4$).{ }^{10}$ All these results are compatible with our findings in univariate analysis that there is a marked effect of cTnI on outcome. However, none of the other studies investigated its additional prognostic value with regard to poor outcome; it proved to be absent in our study. We found no other studies investigating the additional effect of an abnormal cTnI on the occurrence of cardiac and pulmonary complications.

We defined a cTnI value above $0.3 \mu \mathrm{g} /$ litre as abnormal. Other investigators have shown that even minimum peak values (just above detectable value) of troponin can reflect serious cardiac dysfunction. ${ }^{29}$ The severe cardiac dysfunction associated with only small concentrations of cTnI probably explains the lack of a dose dependent effect of an increased concentration of cTnI.

In conclusion, a raised concentration of cTnI is an independent predictor of secondary cardiac and pulmonary complications after SAH. Therefore, cTnI can be used as a marker to identify patients who are at risk of developing cardiopulmonary complications. These patients need special attention for haemodynamic complications and could benefit from early invasive haemodynamic monitoring to guide fluid treatment and inotropic support. Further studies are needed to assess the effectiveness of early invasive monitoring in patients with raised cTnI concentrations on admission.

\section{Authors' affiliations}

W J Schuiling, J Th J Tans, Department of Neurology, Medical Centre Haaglanden, Westeinde Hospital, 2512 VA the Netherlands P J W Dennesen, Department of Intensive Care, Medical Centre Haaglanden

L M Kingma, Department of Radiology, Medical Centre Haaglanden G J E Rinkel, Department of Neurology, University Medical Centre Utrecht, Utrecht, 3584 CX The Netherlands

A Algra, Rudolf Magnus Institute of Neuroscience, and the Julius Centre for Health Sciences and Primary Care, University Medical Centre Utrecht

Dr Rinkel is an established clinical investigator of the Netherlands Heart Foundation (grant D98.014).

Competing interests: none declared

Ethics approval: In this prospective study recording data after routine treatment and no extra patient visiting, submission to our local ethics committee was not required.

\section{REFERENCES}

1 Hop JW, Rinkel GJ, Algra A, et al. Case-fatality rates and functional outcome after subarachnoid haemorrhage: a systematic review. Stroke 1997;28:660-4. 
2 Kassell NF, Torner JC, Clark Haley E, et al. The international cooperative study on the timing of aneurysm surgery. Part I: overall management results. J Neurosurg 1990;73:18-36.

3 Solenski NJ, Haley EC, Kassell NF, et al. Medical complications of aneurysmal subarachnoid haemorrhage: a report of the multicenter, cooperative aneurysm study. Crit Care Med 1995;23:1007-17.

4 Sato K, Masuda T, Izumi T. Subarachnoid haemorrhage and myocardial damage. Clinical and experimental studies. Jpn Heart J 1999;40:683-701.

5 Macmillan CSA, Grant IS, Andrews PJD. Pulmonary and cardiac sequelae of subarachnoid haemorrhage: time for active management? Intensive Care Med 2002; 28:1012-23.

6 Naredi S, Lambert G, Edén E, et al. Increased sympathetic nervous activity in patients with nontraumatic subarachnoid haemorrhage. Stroke 2000;31:901-6.

7 Antman EM, Tanasijevic MJ, Thompson B, et al. Cardiac-specific troponin I levels to predict the risk of mortality in patients with acute coronary syndromes. N Engl J Med 1996;335:1342-9.

8 Turner A, Tsamitros M, Bellomo R. Myocardial cell injury in septic shock. Crit Care Med 1999:27:1775-80.

9 Tung P, Kopelnik A, Banki N, et al. Predictors of neurocardiogenic injury after subarachnoid haemorrhage. Stroke 2004;35:548-53.

10 Claassen J, Vu A, Kreiter KT, et al. Effect of acute physiologic derangements on outcome after subarachnoid haemorrhage. Crit Care Med 2004;32:832-8.

11 Parekh N, Venkatesh B, Cross D, et al. Cardiac troponin I predicts myocardial dysfunction in aneurysmal subarachnoid haemorrhage. J Am Coll Cardiol 2000;36:1328-35.

12 Deibert E, Barzilai B, Braverman A, et al. Clinical significance of elevated troponin I levels in patients with nontraumatic subarachnoid haemorrhage. J Neurosurg 2003;98:741-6.

13 Kassell NF, Torner JC, Clark Haley E, et al. The international cooperative study on the timing of aneurysm surgery. Part I: overall management results. J Neurosurg 1990;73:18-36.

14 Hijdra A, van Gijn J, Nagelkerke N, et al. Prediction of delayed cerebral ischemia, rebleeding and outcome after aneurysmal subarachnoid hemorrhage. Stroke 1988;19:1250-6.

15 Drake CG. Report of World Federation on Neurological Surgeons committee on a universal subarachnoid haemorrhage grading scale. J Neurosurg 1988;68:985-6.

16 Hijdra A, Brouwers PJ, Vermeulen M, et al. Grading the amount of blood on computed tomograms after subarachnoid haemorrhage. Stroke 1990;21:1156-61.
17 Bernard GR, Artigas A, Brigham KL, et al. The American-European consensus conference on ARDS: definitions, mechanisms, relevant outcomes, and clinical trial coordination. Am J Respir Crit Care Med 1994; 149:818-24.

18 Pugin J, Auckenthaler R, Lew DP, et al. Diagnosis of ventilator-associated pneumonia by bacteriologic analysis of bronchoscopic and nonbronchoscopic "blind" bronchoalveolar lavage fluid. Am Rev Respir Dis 1991;143:1121-9.

19 Members of the American College of Chest Physicians. American college of chest physicians/society of critical care medicine consensus conference: definitions for sepsis and organ failure and guidelines for the use of innovative therapies in sepsis. Crit Care Med 1992;20:864-74.

20 Stoutenbeek CP, van Saene HK, Miranda DR, et al. The effect of selective decontamination of the digestive tract on colonisation and infection rate in multiple trauma patients. Intensive Care Med 1984;10:185-92.

21 Van Swieten JC, Koudstaal PJ, Visser MC, et al. Interobserver agreement for the assessment of handicap in stroke patients. Stroke 1988;19:604-7.

22 Hanley JA, McNeil BJ. The meaning and use of the area under a receiver operating characteristic (ROC) curve. Radiology 1982;7:37-43.

23 Doshi R, Path MRC, Neil-Dwyer G. A clinicopathological study of patients following a subarachnoid haemorrhage. J Neurosurg 1980;52:295-301.

24 Masuda T, Sato K, Yamamoto S, et al. Sympathetic nervous activity and myocardial damage immediately after subarachnoid haemorrhage in a unique animal model. Stroke 2002;33:1671-6.

25 Deehan SC, Grant IS. Hemodynamic changes in neurogenic pulmonary oedema: effect of dobutamine. Intensive Care Med 1996;22:672-6.

26 Porchet F, Chiolero R, de Tribolet N. Hypotensive effect of nimodipine during treatment for aneurysmal subarachnoid haemorrhage. Acta Neurochir (Wien) 1995; 137:62-9.

27 Brun-Buisson C, Doyon F, Carlet J. Bacteremia and severe sepsis in adults: a multicenter prospective survey in ICUs and wards of 24 hospitals. French bacteremia-sepsis study group. Am J Respir Crit Care Med 1996; 154:617-24.

28 Horowitz MB, Willet D, Keffer J. The use of cardiac troponin-I (cTnl) to determine the incidence of myocardial ischemia and injury in patients with aneurysmal and presumed aneurysmal subarachnoid haemorrhage. Acta Neurochir (Wien) 1998;140:87-93.

29 Bulsara KR, McGirt MJ, Liao L, et al. Use of peak troponin value to differentiate myocardial infarction from reversible neurogenic left ventricular dysfunction associated with aneurysmal subarachnoid haemorrhage. J Neurosurg 2003;98:524-8. 